\title{
Accidentally-on-purpose: findings from a qualitative study exploring pregnancy intention and long-acting reversible contraceptive use
}

\author{
Jacqueline Coombe, Melissa L Harris, Deborah Loxton
}

Research Centre for Generational Health and Ageing, Faculty of Health and Medicine, University of Newcastle, Callaghan, New South Wales, Australia

\section{Correspondence to}

Jacqueline Coombe, Research Centre for Generational Health and Ageing, Faculty of Health and Medicine, University of Newcastle, University Drive, Callaghan, NSW 2308, Australia; jacqueline.coombe@uon.edu.au

Received 21 March 2018 Revised 30 April 2018 Accepted 23 May 2018
(D) Check for updates

To cite: Coombe J, Harris ML, Loxton D. BMJ Sex Reprod Health 2018;44:207-213.

\begin{abstract}
Background Although it is known that pregnancy intention impacts contraceptive use, there has been little exploration into the relationship between pregnancy intention and long-acting reversible contraception (LARC) nonuse in the Australian context.

Methods Semi-structured telephone interviews with a sample of participants from the Contraceptive Use, Pregnancy Intention and Decisions (CUPID) Study were conducted in 2016.

Results Of the 59 women contacted, 15 participated in an interview. One theme arising from these interviews is reported here. Results from the analysis suggest that women with ambivalent or unclear plans toward pregnancy were less likely to perceive LARC as a suitable method for them. Conversely, women who clearly intended to avoid pregnancy and who had clear plans for future pregnancy valued these methods, and often framed their future plans for pregnancy within the context of their chosen LARC.

Conclusions Findings presented demonstrated the complex relationship between pregnancy intention and contraceptive use. In particular, this study provided insight into the complex notion of pregnancy ambivalence. Dichotomous definitions of pregnancy as intended or unintended were found to be inadequate in encapsulating actual reproductive experiences.
\end{abstract}

\section{INTRODUCTION}

Unintended pregnancy, and how to prevent them, is a much discussed topic in the sexual and reproductive health literature. Many a paper begins with a sentence about rates of unintended pregnancy in a country or population of interest (for example ${ }^{1}$ ), and these experiences are often equated with negative health

\section{Key messages}

- Women with ambivalent attitudes were reluctant to use LARC, potentially because these methods do not allow for 'user-error'.

- Women with clear, future plans for pregnancy valued longer-acting methods and often framed their plans for pregnancy within the context of their chosen LARC.

- Dichotomous definitions of pregnancy 'intention' are unable to capture the complexity of women's reproductive experiences.

outcomes for women and their children. ${ }^{3}$ Although unintended pregnancy and contraceptive use, or a lack thereof, are undeniably linked, the relationship between the two is complex. Studies show that women often report using some form of contraception at the time of unintended pregnancy, ${ }^{4}$ while others demonstrate a lack of, or inconsistent, use of contraceptives in the presence of pregnancy ambivalence. ${ }^{5}$

Long-acting reversible contraception (LARC) encompasses highly effective, long-lasting contraceptive methods, which include intrauterine devices (IUDs) and contraceptive implants. Both methods are placed into the body by a healthcare professional, are very effective for years at a time and cannot be removed without the aid of a healthcare provider. ${ }^{6}$ Given their effectiveness, these methods are promoted for their ability to prevent unintended pregnancy. ${ }^{78}$ However, rates of use among young Australian women, who have reportedly high rates of unintended pregnancy, are low. ${ }^{4} 910$ Data from the 
Second Australian Study of Health and Relationships, for example, shows high rates of pill and condom use among women aged $20-29$ years $(49.1 \%$ and $37.6 \%$, respectively) as compared with use of IUDs (2.0\%) and the contraceptive implant (3.9\%). ${ }^{11}$ Although increases in LARC use have been seen in Australia over the past 10 years, this increase has mostly occurred among older women who are increasingly using LARC instead of sterilisation. ${ }^{11}$ While there are many reasons women may choose to use, or not use, LARC, including their potential side effects and fears about their safety, ${ }^{12} 13$ there has been little exploration into the relationship between pregnancy intention and LARC non-use in the Australian context, despite evidence suggesting the impact of pregnancy intention on LARC (non-)use internationally. ${ }^{14}$

This article reports on the findings from qualitative interviews conducted with women aged between 20 and 27 years, which aimed to understand low rates of LARC use among young Australian women. This article focuses on one theme arising from these interviews, namely the relationship between pregnancy intention and LARC non-use.

\section{METHODS}

The findings presented here form part of a larger project which utilised the Contraceptive Use, Pregnancy Intention and Decisions (CUPID) Study to explore non-use of LARC. In brief, CUPID is a longitudinal, population-based cohort study which recruited 3795 women aged 18-23 years at baseline in 2012-2013. Participants were recruited into the cohort using a number of online and offline methods, of which Facebook recruitment was most successful. Participant demographics were monitored against the Australian Census to ensure the sample was broadly representative of the larger population. Participants were surveyed online a total of three times, with the study concluding in early 2016. Further details about CUPID have been published elsewhere. ${ }^{15}$ The current study comprised semi-structured telephone interviews with a sample of CUPID participants to further understand their contraceptive practices and LARC non-use. Semi-structured telephone interviews were chosen for their flexibility in regards to data collection ${ }^{16}$ and recruitment, ${ }^{17}$ as CUPID participants were located across Australia.

\section{Recruitment}

Recruitment and data collection were conducted in 2016. Potential participants had completed all three CUPID surveys and had supplied an email address. Women were randomly drawn from the CUPID database by a data assistant in batches of 10 to allow recruitment, data collection and preliminary analysis to proceed iteratively. These women were sent an email by the first author explaining the sub-study and inviting them to participate. Non-responders were sent a reminder email at 1 and 2 weeks after the initial invitation email; no further attempt at contact was made after the second reminder email. Participants who replied to an invitation email were contacted by the researcher via return email to confirm their interest, to ask if they had any questions and to organise a time for an interview. Recruitment concluded once data saturation had been reached. This study was not representative of the entire population of interest; however, via purposive sampling, women relevant to the research question were interviewed and their experiences provided insight into the phenomenon of interest. ${ }^{16}$ Interviews were conducted by the first author. All interviews were recorded using a voice recorder and telephone pickup device and were conducted with a semi-structured interview schedule. Interviews lasted between $30 \mathrm{~min}$ and 1 hour, and were transcribed verbatim in accordance with suggestions made by Braun and Clarke. ${ }^{18}$

\section{Data analysis}

A thematic analysis approach outlined by Braun and Clarke $^{18}$ was utilised to analyse the interview data. In brief, this process involves familiarisation with the data, initial coding, searching for themes, reviewing themes, defining and naming themes, and producing the report. The analysis was conducted by the first author with input from the second and third authors throughout. Data saturation (where no new substantive themes in relation to the research question were being developed) was assessed during the initial coding phase of analysis. Interrater-reliability was used to facilitate data saturation; all authors met throughout the data collection and initial analysis phases to discuss the emerging themes and all agreed that data saturation had been reached. ${ }^{19}$ NVivo qualitative analysis software was utilised to facilitate analysis. ${ }^{20}$ This study took a broadly social constructionist approach, aiming to locate contraceptive practice and pregnancy intention within the social context while simultaneously recognising the lived experiences of these practices. The guidelines proposed by Kitto and colleagues ${ }^{19}$ were utilised to ensure quality and rigour in this study.

\section{Ethics approval}

The CUPID Study received ethical approval from the University of Newcastle (H-2011-0331), the University of Queensland (2011001055), Family Planning NSW (R2011-05) and the Australian Government Department of Health (31/2013). A variation to the original University of Newcastle ethical approval to conduct interviews with a sample of CUPID participants was sought and approved in November 2015, prior to the commencement of this sub-study.

\section{Patient and public involvement}

Patients were not involved in this study. With regards to the CUPID Study, participant feedback was regularly 
integrated into revisions of the survey, and a reference group of women the same age as study participants advised on recruitment and survey development at the beginning of the project. Interview participants who asked to be informed about the findings from this sub-study were provided with a summary of results at the conclusion of the project.

\section{RESULTS}

Of the 59 women invited to participate via email, 21 responded. One potential participant responded to the email more than a year after the final reminder email was sent and was therefore ineligible to participate as the study had been completed by this time. Twenty women were provided with further information about the study. Five women did not respond to this email, nor to a follow-up email. Reasons for non-participation were therefore not collected. In total, 15 women participated in an interview. Seven participants reported past use of either an IUD or contraceptive implant; four were still using these methods at the time of their interview. Three of the participants reported previous pregnancies; one reported one unintended pregnancy, resulting in termination, another reported one unintended and one planned pregnancy, and the third two planned pregnancies. Both participants with children had two each, and were breastfeeding their youngest child at the time of the interview. Further demographic details are shown in table 1 . This article reports on one theme arising from these interviews, namely the relationship between contraceptive practices and pregnancy intention. All participants were given a pseudonym to maintain anonymity.

\section{'Now is not the right time'}

For most of the participants, having a child was not in their immediate plans. That is, 'now' (at least, at the time of their interview) was not the right time to fall pregnant. This understanding of 'not the right time' was framed within the context of when the right time would be, generally after certain 'milestones' such as a particular age, a stable long-term relationship, completion of education, financial security and so on, had been reached. The absence of these milestones meant it was 'not the right time' for pregnancy. However, despite clearly indicating that 'now' was definitely not the right time for pregnancy, some respondents were unsure when the right time would be. For example, Anna said:

Yeah eventually but well I guess I want to finish uni and get some money and maybe think about it. Yeah ideally that would be - ideally the plan is to have kids [children] I guess. [Anna, 22 years old, pill and withdrawal]

Although clear about what milestones they wanted to reach before pregnancy, like Anna, these women did not have a clear idea about when exactly the right
Table 1 Participant demographic information $(n=15)$

\begin{tabular}{|c|c|c|}
\hline Demographic parameters & $n$ & $\%$ * \\
\hline Age (years) (mean) & $20-27(23.2)$ & \\
\hline \multicolumn{3}{|l|}{ Residential status } \\
\hline Major/regional city & 15 & 100.00 \\
\hline Rural/remote & 0 & 0.00 \\
\hline \multicolumn{3}{|l|}{ Employment status } \\
\hline Full-time & 3 & 20.00 \\
\hline Part-time & 4 & 26.66 \\
\hline Casual & 3 & 20.00 \\
\hline Maternity leave & 1 & 6.66 \\
\hline Full-time student & 4 & 26.66 \\
\hline \multicolumn{3}{|l|}{ Highest educationt } \\
\hline University degree & 13 & 86.66 \\
\hline TAFE qualification & 1 & 6.66 \\
\hline Completed Year 12 & 1 & 6.66 \\
\hline \multicolumn{3}{|l|}{ Access to health services } \\
\hline Always easy & 12 & 80.00 \\
\hline Usually easy & 3 & 20.00 \\
\hline \multicolumn{3}{|l|}{ Distance to health services } \\
\hline$<10 \mathrm{~km}$ & 15 & 100.00 \\
\hline$>10 \mathrm{~km}$ & 0 & 0.00 \\
\hline \multicolumn{3}{|l|}{ Relationship status } \\
\hline In a relationship & 14 & 93.33 \\
\hline Single & 1 & 6.66 \\
\hline \multicolumn{3}{|l|}{ Children (n) } \\
\hline 0 & 13 & 86.66 \\
\hline 1 & 0 & 0.00 \\
\hline 2 & 2 & 13.33 \\
\hline \multicolumn{3}{|l|}{ Current contraception } \\
\hline Pill only & 3 & 20.00 \\
\hline Pill and condoms & 2 & 13.33 \\
\hline Pill and withdrawal & 1 & 6.66 \\
\hline Hormonal IUD‡ & 2 & 13.33 \\
\hline Implant $\ddagger$ & 2 & 13.33 \\
\hline Condoms and natural family planning & 1 & 6.66 \\
\hline Condoms only & 1 & 6.66 \\
\hline Breastfeeding and withdrawal & 1 & 6.66 \\
\hline Breastfeeding and abstinence & 1 & 6.66 \\
\hline Nothing & 1 & 6.66 \\
\hline
\end{tabular}

* Rounded to $2 \mathrm{dps}$.

tHas completed or is currently completing.

¥In total, four participants (26.66\%) were using long-acting reversible contraception (LARC) at the time of the interview.

IUD, intrauterine device; TAFE, Technical and Further Education.

time would be. In contrast, others, particularly those using a LARC, articulated more specific timing-based plans for pregnancy. These plans were often contextualised within the effective timeframe for their specific 
method. For example, Eliza, who was using a hormonal IUD, said:

...I probably don't want to have kids for another about 5 years so I think yeah, another Mirena [hormonal IUD] would get me to that point. [Eliza, 25 years old, hormonal IUD]

Comparably, Jane felt the implant provided her with a more feasible timeline to bring her to a time when she and her partner would be ready for children. She reported deciding not to use a hormonal IUD because she felt it was too long-lasting, and that she would have to have it removed "reasonably quickly". Given that LARC has a very clear end date, it appears that women who use these methods are prompted to have clearer plans regarding their future fertility, because these plans are, at least in part, dependant on the lifetime of their LARC.

\section{'Okay either way'}

Although none of the women reported actively trying to conceive at the time of the interviews, some women clearly reported ambivalent feelings towards pregnancy. Although pregnancy ambivalence has been defined as unclear or uncertain feelings towards pregnancy, ${ }^{521}$ this was not congruent with the experiences reported by the women in this study. Rather, pregnancy ambivalence was an acknowledged, clear stage of their reproductive lives. For example, Claire spoke about what she thought she and her partner would do if they experienced an unintended pregnancy:

... if we accidently fell pregnant we acknowledge it wouldn't be a total disaster cause we are both sort of at a point in our lives where we could deal with that um and that we would keep the child um as opposed to perhaps how I felt like 6 years ago when I was 18 ... [Claire, 24 years old, condoms]

Although clearly noting that now is not the ideal time for pregnancy, Claire felt that she and her partner could "deal with that" and would continue the pregnancy. These ambivalent feelings towards pregnancy were often spoken about in combination with contraceptive practices that were perhaps not as strict as they had once been. For example, Emma spoke about a time when she wasn't being particularly careful to take her pill properly, and her rationalisation for this decision:

...I remember there was one where like I had forgotten to take it, the first pill after my period which is bad...so I did that but even then I didn't abstain...cause I was like oh it will be fine [laughs] and because the current boyfriend I am with now like its I mean I'm 99\% sure that you know we will you know get married and have kids [children] one day so and I'm sort of at that age now... if I did get pregnant I would 100\% keep the child whereas you know back in you know my other relationship when I was younger it wouldn't have been the case so yeah. [Emma, 25 years old, the Pill]
Like Emma, others also reported transitioning into pregnancy ambivalence and relaxing their contraceptive practices. Kate said:

...if it happens then it is obviously supposed to happen and we are not, not preventing it but we are not exactly aiming for it either. If that makes sense.

[Kate, 27 years old, breastfeeding and withdrawal]

She went on to say "I feel quite irresponsible by saying it out loud!" Contrary to suggestions that ambivalence represents unclear feelings towards pregnancy, these comments suggest that being 'okay either way' with pregnancy characterises a specific time in the lives of women. In addition, these women were generally using a contraceptive method that accommodated 'room for error' and were less concerned about instances of possible contraceptive 'failure'.

\section{'Maybe never'}

A third group of women expressed uncertainty towards childbearing. For example, although Hannah had obviously thought about when the right time to have children would be, she was unsure whether or not she would have children. She said:

Um probably not for a while yet. I've got, I want to graduate and I want to go um to med [medical] school after nursing so yeah I probably want to get all that done first, before I even thought about kids. I don't really like kids to be honest though so I am not sure if it will be on the cards or not. [Hannah, 20 years old, the Pill]

Zoe, on the other hand, clearly did not want to ever have children of her own. She explained:

...I've known my whole life that I don't want to have children...no one's letting me do it [access sterilisation] ...they all seem to think I will regret it one day. [Zoe, 22 years old, the Pill and condoms as needed]

Although using the Pill at the time of the interview, Zoe was seeking to obtain a copper IUD, which she intended to use until she could access sterilisation. While Zoe appeared to consider the efficacy of LARC as a way to meet her reproductive goals, both Hannah and others were using short-term, less effective methods to protect themselves during periods of sexual activity.

\section{DISCUSSION}

In contrast to previous research which defines pregnancy ambivalence as unclear, contradictory or incongruent attitudes towards pregnancy, ${ }^{521}$ for the women in the current study, pregnancy ambivalence was a clear phase in their reproductive lives. While the relationship between pregnancy ambivalence and inconsistent contraceptive use is known, ${ }^{5}$ the current study adds to this understanding by exploring the experiences behind this inconsistent use, where instances 
of contraceptive failure were met with less concern, and contraceptive effectiveness appeared not to drive method choice. Further, as has been reported internationally, ${ }^{14}$ women with ambivalent attitudes towards pregnancy were less likely to consider LARC as suitable for them, potentially because these methods do not allow 'accidents' to occur, and are thus incongruent with their reproductive desires. Perhaps using contraception, although somewhat inconsistently, allows women to engage in socially expected behaviours (ie, using contraception in the absence of clearly planning pregnancy), while simultaneously subverting these norms (ie, 'unintended' pregnancy while using some form of contraception). Further investigation is, however, necessary.

Women with clear current pregnancy avoidance plans, but unclear ideas about when exactly to have children, were also found to be reluctant to use LARC. This may have something to do with the nature of LARC itself, whose longevity and strict effectiveness timeframe may make women feel forced into making firm decisions about their plans for pregnancy. Although LARC can be removed early, a lack of knowledge about these methods may mean some women are unaware of this possibility. Indeed, misperceptions regarding LARC are consistently cited as a barrier to use, ${ }^{22}$ particularly among young people. ${ }^{23}{ }^{24}$ Women who are unwilling to make a decision about their future pregnancy plans may therefore perceive LARC to be undesirable, despite clearly intending to avoid pregnancy in the present, as has also been reported elsewhere. ${ }^{25}$ Conversely, women with clear current pregnancy avoidance plans, and clear future plans for pregnancy, perceived LARC as most suited to their needs, and these women articulated their future pregnancy plans in relation to the effectiveness timeframe of their chosen method.

Finally, although most of the women in this study spoke about when they would have children, rather than if, there were a small group of women who reported potentially never wanting to reproduce. Although remaining 'voluntarily childless' in a society which values motherhood has its own challenges, ${ }^{26} 27$ for one participant these challenges manifested in an inability to access sterilisation. Although alone in this dataset, this experience has been reported elsewhere, where women report that their request for sterilisation is not taken seriously by their doctor, ${ }^{28}$ or report being discriminated against for their choice to remain childless. ${ }^{26}$

Decisions regarding contraceptive use and intentions towards pregnancy do not exist in a vacuum and are both influenced by a myriad of factors (see for example $\left.\mathrm{e}^{12-14}\right)$. Without discounting these often complex factors, the findings presented here add to the existing literature by examining LARC (non-)use in the context of pregnancy intention and have implications not only for LARC use, but also more broadly the reproductive experiences of women. First, acknowledging that not all women desire a planned or well-timed pregnancy ${ }^{29} 30$ (or even, any pregnancy at all) is essential. Supporting women's contraceptive decisions (to not use LARC, or requesting early removal, for example), is essential to providing high-quality contraceptive care $^{31}$ and reproductive autonomy. ${ }^{32}$ Further, these findings contribute to the growing body of work that highlights the inadequacy of dichotomous conceptualisations of pregnancy as either intended or unintended in capturing women's actual reproductive experiences (see for example ${ }^{30}$ ). A move towards encompassing the complexity of these experiences is essential, although this may pose unique challenges to providing preconception care. How best to provide this care to women with ambivalent pregnancy attitudes, without assuming that all women want or desire children, warrants further investigation.

\section{Study strengths and limitations}

A key limitation of this study was its recruitment strategy. In particular, due to the nature of the recruitment strategy, reasons for non-participation were not collected and the differences between women who agreed to participate and those who did not respond to our invitation email were not able to be examined. Further, our recruitment strategy yielded a sample that was relatively homogenous, and our findings should be interpreted within this context. However, despite these limitations, results presented here demonstrated the complex and varied experiences of pregnancy intention and its relation to LARC (non-)use. These findings shed light on a currently under examined aspect of LARC uptake in the Australian context and set the framework for future research in this area.

\section{CONCLUSIONS}

Without overgeneralising, the findings presented shed light on the complex relationship between pregnancy intention and contraceptive practices. In particular, women with ambivalent or unclear plans toward pregnancy were found to be less likely to perceive LARC as suitable for them, while women with clear pregnancy avoidance plans valued these methods.

Acknowledgements The authors would like to thank the women who kindly shared their experiences with them.

Contributors MLH and DL were investigators on the initial CUPID Study. JC, MLH and DL devised the design of the substudy and JC conducted the interviews, analysed the resultant data and drafted the manuscript. MLH and DL supervised the study progress, assisted with data analysis and provided intellectual input into the paper. All authors read and approved the manuscript prior to submission.

Funding JC is a PhD student supported by an Australian Government Research Training Program (RTP) Scholarship. The authors gratefully acknowledge funding support from the Australian Research Council (Linkage Project Grant LP100200349), and industry partners Family Planning NSW 
and Bayer Australia Ltd. MLH was previously supported by the Australian Research Council through the above Linkage Project Grant and DL was a chief investigator.

Competing interests $\mathrm{JC}$ is a $\mathrm{PhD}$ student supported by an Australian Government Research Training Program (RTP) Scholarship. The research on which this paper is based was supported under the Australian Research Council's Linkage Projects funding scheme (Project Number LP100200349). MLH was previously supported by the Australian Research Council through the above Linkage Project grant which involved some cash and in-kind support from the Partner Organisations, Family Planning NSW and Bayer Australia Ltd. DL was a chief investigator on this project.

Patient consent Not required.

Ethics approval University of Newcastle Human Research Ethics Committee.

Provenance and peer review Not commissioned; externally peer reviewed.

Data sharing statement There is no additional unpublished data from this qualitative study.

(C) Article author(s) (or their employer(s) unless otherwise stated in the text of the article) 2018. All rights reserved. No commercial use is permitted unless otherwise expressly granted.

\section{REFERENCES}

1 Aztlan-James EA, McLemore M, Taylor D. Multiple unintended pregnancies in US women: a systematic review. Women's Health Issues 2017;27:407-13.

2 Birgisson NE, Zhao Q, Secura GM, et al. Preventing unintended pregnancy: the contraceptive CHOICE project in review. J Womens Health 2015;24:349-53.

3 Blumenthal PD, Voedisch A, Gemzell-Danielsson K. Strategies to prevent unintended pregnancy: increasing use of long-acting reversible contraception. Hum Reprod Update 2011;17:dmq026.

4 Coombe J, Harris ML, Wigginton B, et al. Contraceptive use at the time of unintended pregnancy: findings from the Contraceptive Use, Pregnancy Intention and Decisions study. Aust Fam Physician 2016;45:842-8.

5 Schwarz EB, Lohr PA, Gold MA, et al. Prevalence and correlates of ambivalence towards pregnancy among nonpregnant women. Contraception 2007;75:305-10.

6 Family Planning Alliance Australia. Efficacy of contraception methods. 2015 http://familyplanningallianceaustralia.org.au/ larc/ (accessed 14 Aug 2015).

7 American College of Obstetricians and Gynecologists Committee Opinion. Increasing access to contraceptive implants and intrauterine devices to reduce unintended pregnancy. 2015 http://www.acog.org/Resources-AndPublications/Committee-Opinions/Committee-on-GynecologicPractice/Increasing-Access-to-Contraceptive-Implants-andIntrauterine-Devices-to-Reduce-Unintended-Pregnancy (accessed 29 Oct 2015).

8 Mazza D, Bateson D, Frearson M, et al. Current barriers and potential strategies to increase the use of long-acting reversible contraception (LARC) to reduce the rate of unintended pregnancies in Australia: an expert roundtable discussion. Aust N Z J Obstet Gynaecol 2017;57:206-12.

9 Coombe J, Harris ML, Loxton D. Who uses long-acting reversible contraception? Profile of LARC users in the CUPID cohort. Sex Reprod Healthc 2017;11:19-24.

10 Rassi A, Wattimena J, Black K. Pregnancy intention in an urban Australian antenatal population. Aust N Z J Public Health 2013;37:568-73.
11 Richters J, Fitzadam S, Yeung A, et al. Contraceptive practices among women: the second Australian study of health and relationships. Contraception 2016;94:548-55.

12 Payne JB, Sundstrom B, DeMaria AL. A qualitative study of young women's beliefs about intrauterine devices: fear of infertility. J Midwifery Womens Health 2016;61:482-8.

13 Spies EL, Askelson NM, Gelman E, et al. Young women's knowledge, attitudes, and behaviors related to long-acting reversible contraceptives. Womens Health Issues 2010;20:3949.

14 Higgins JA. Pregnancy ambivalence and long-acting reversible contraceptive (LARC) use among young adult women: a qualitative study. Perspect Sex Reprod Health 2017;49:14956.

15 Harris ML, Loxton D, Wigginton B, et al. Recruiting online: lessons from a longitudinal survey of contraception and pregnancy intentions of young Australian women. Am J Epidemiol 2015;181:737-46.

16 Bryman A. Social research methods. Oxford: Oxford University Press, 2008.

17 Trier-Bieniek A. Framing the telephone interview as a participant-centred tool for qualitative research: a methodological discussion. Qual Res 2012;12:630-44.

18 Braun V, Clarke V. Using thematic analysis in psychology. Qual Res Psychol 2006;3:77-101.

19 Kitto SC, Chesters J, Grbich C. Quality in qualitative research. Med J Aust 2008;188:243-6.

20 QSR International Pty Ltd. NVivo qualitative data analysis software. Version 10: ed. QSR International Pty Ltd, 2012.

21 Higgins JA, Popkin RA, Santelli JS. Pregnancy ambivalence and contraceptive use among young adults in the United States. Perspect Sex Reprod Health 2012;44:236-43.

22 Black KI, Bateson D, Harvey C. Australian women need increased access to long-acting reversible contraception. Med J Aust 2013;199:317-8.

23 Garrett CC, Keogh LA, Kavanagh A, et al. Understanding the low uptake of long-acting reversible contraception by young women in Australia: a qualitative study. BMC Womens Health 2015;15:72.

24 Ritter T, Dore A, McGeechan K. Contraceptive knowledge and attitudes among 14-24-year-olds in New South Wales, Australia. Aust N Z J Public Health 2015;39:267-9.

25 Sundstrom B, Ferrara M, DeMaria AL, et al. Integrating pregnancy ambivalence and effectiveness in contraceptive choice. Health Commun 2017;32:1-8.

26 Doyle J, Pooley JA, Breen L. A phenomenological exploration of the childfree choice in a sample of Australian women. $J$ Health Psychol 2013;18:397-407.

27 Rich S, Taket A, Graham M, et al. 'Unnatural', 'Unwomanly', 'uncreditable' and 'undervalued': the significance of being a childless woman in Australian society. Gender Issues 2011;28:226-47.

28 Gillespie R. When no means no: disbelief, disregard and deviance as discourses of voluntary childlessness. Women's Studies International Forum 2000;23:223-34.

29 Borrero S, Nikolajski C, Steinberg JR, et al. "It just happens": a qualitative study exploring low-income women's perspectives on pregnancy intention and planning. Contraception 2015;91:150-6.

30 Aiken AR, Borrero S, Callegari LS, et al. Rethinking the pregnancy planning paradigm: unintended conceptions or unrepresentative concepts? Perspect Sex Reprod Health 2016;48:147-51. 
31 Strasser J, Borkowski L, Couillard M, et al. Access to removal of long-acting reversible contraceptive methods Is an essential component of high-quality contraceptive care. Womens Health Issues 2017;27:253-5.
32 Gomez AM, Fuentes L, Allina A. Women or LARC first? Reproductive autonomy and the promotion of long-acting reversible contraceptive methods. Perspect Sex Reprod Health 2014;46:171-5. 